\title{
Interkulturelles Coaching für geflüchtete Fachkräfte in Gesundheitsberufen: Entwicklung, Durchführung und Evaluation
}

\author{
Sidra Khan-Gökkaya • Mike Mösko
}

Online publiziert: 23. Juli 2020

(C) Der/die Autor(en) 2020

Zusammenfassung Geflüchtete Fachkräfte erleben verschiedene Barrieren auf dem Arbeitsmarkt, von denen einige in einem Interkulturellen Coaching adressiert werden können. Dieser Artikel beleuchtet die Entwicklung, Durchführung und Evaluation eines Interkulturellen Coachings für geflüchtete Personen in Gesundheitsberufen. Die Entwicklung und Erprobung des Coachings erfolgte stufenweise. Um das Coaching zu evaluieren, wurde ein multimethodales Studiendesigns gewählt. Die Ergebnisse zeigen, dass das Coaching nicht nur für die Integration in den Beruf, sondern generell für den Aufenthalt in Deutschland als hilfreich angesehen wird.

Schlüsselwörter Interkulturelles Coaching $\cdot$ Geflüchtete $\cdot$ Gesundheitsberufe

\section{Cross-cultural coaching for refugee health professionals: development, implementation and evaluation}

\begin{abstract}
Refugee health professionals experience several barriers on their path to re-integration. Some of these barriers may be addressed through a cross-cultural coaching. This article focuses on the development, implementation and evaluation of a cross-cultural coaching. The coaching was developed and implemented step by step. A multi-method study design was chosen to evaluate the coaching. Results indicate that coaching is not only seen as useful for integration into the profession, but also for their situation in Germany.
\end{abstract}

Keywords Cross-cultural coaching $\cdot$ Refugees $\cdot$ Health professionals

S. Khan-Gökkaya $(\bowtie) \cdot$ Prof. Dr. M. Mösko

Universitätsklinikum Hamburg-Eppendorf (UKE), Martinistraße 52, 20246 Hamburg, Deutschland

E-Mail: s.khan-goekkaya@uke.de

Prof. Dr. M. Mösko

E-Mail: mmoesko@uke.de 


\section{Hintergrund}

Die Zahl der Personen, die nach Europa und Deutschland flüchten mussten, ist seit 2014 angestiegen und seit 2017 stark zurückgegangen (Bundesamt für Migration und Flüchtlinge 2020, S. 6). Dennoch ist das Thema Fluchtmigration in der öffentlichen und wissenschaftlichen Debatte zentral (Aden et al. 2019, S. 303). Für die erfolgreiche gesellschaftliche Integration der geflüchteten Personen in Deutschland ist der Zugang zum Arbeitsmarkt ein wesentlicher Schlüssel. Geflüchtete Personen unterliegen jedoch verschiedenen Einschränkungen am Arbeitsmarkt. Aufenthaltsrechtliche Bestimmungen grenzen den Zugang zum Arbeitsmarkt sowie zu Sprachkursen ein und erschweren damit den Erwerb der (Fach-) Sprache und einen Einblick in relevante Berufsfelder (Johansson 2016, S. 21).

Geflüchtete Fachkräfte, die in Gesundheitsberufen arbeiten wollen, müssen zusätzliche Hürden überwinden. Da Gesundheitsberufe in Deutschland gesetzlich und berufsrechtlich reglementiert sind, müssen geflüchtete Fachkräfte einen Anerkennungsprozess durchlaufen und sehr gute Sprachkenntnisse nachweisen (Bundesamt für Migration und Flüchtlinge 2011, S. 26ff.). Der Anerkennungsprozess wird allerdings als langwierig und herausfordernd beschrieben (Desiderio 2016, S. 2). Weitere Barrieren stellen das fehlende Wissen über Karrierewege (Cohn et al. 2006, S. 75) und Strategien zur Jobsuche (Willott und Stevenson 2013, S. 127) dar sowie eine fehlende Vertrautheit mit dem Gesundheitssystem des Landes (Ong et al. 2004, S. 32). Durch die Flucht können Menschen einen Bruch in ihrer Arbeitsbiographie (Etzold 2017, S. 337) und den Verlust ihrer beruflichen Identität (Colic-Peisker und Tilbury 2003, S. 68) erleben. Eine Ent-Qualifizierung (Stewart 2003, S. 9), der Verlust von Selbstbewusstsein (Jirovsky et al. 2015, S. 7) und weitere negative psychische Auswirkungen (Cohn et al. 2006, S. 75) können die Folge sein. Das Arbeiten unter prekären Bedingungen (Diekmann und Fereidooni 2019, S. 349) und die Erfahrung von rassistischer Diskriminierung (Jirovsky et al. 2015, S. 8) am Arbeitsplatz erschweren eine gleichberechtigte Teilhabe am Arbeitsmarkt.

Studien im Kontext der Arbeitsmarktintegration von ausländischen Fachkräften in Gesundheitsberufe in Deutschland berichten von ähnlichen Barrieren, wie sie geflüchtete Personen erleben. Exemplarisch skizziert eine Studie die Erfahrungen von Ärzten und Ärztinnen mit Migrationshintergrund eine Bandbreite an Herausforderungen in drei Bereichen (Klingler und Marckmann 2016, S. 5). Der erste Bereich bezieht sich auf organisationale Schwierigkeiten. Dazu zählen mangelnde Unterstützung sowie mangelnde Entwicklungsmöglichkeiten innerhalb der Organisationen und ungleiche Behandlung. Der zweite Bereich bezieht sich auf eigene erlebte Schwierigkeiten, z. B. der Erwerb der Sprache und das Wissen über das Gesundheitssystem. Der dritte Bereich bezieht sich auf Schwierigkeiten im interpersonellen Bereich mit Kolleg/innen und Patient/innen. Der Kontakt zu Kolleg/innen wird im Rahmen einer weiteren Studie zur Integration ausländischer Pflegekräfte als besonders herausfordernd erlebt (Pütz et al. 2019, S. 73). Konflikte zwischen zugewanderten und nicht-zugewanderten Fachkräften treten nicht selten auf, da nichtzugewanderte Fachkräfte Informationen zurückhalten oder unverständlich erklären, die für einen reibungslosen Ablauf notwendig wären (ebd., S. 159). Die Integration von ausländischen Pflegekräften auf Station wird dadurch erschwert, und das 
Konfliktpotential steigt (ebd., S. 159). Gleichzeitig führt die mangelnde Vertrautheit mit dem hiesigen Gesundheitssystem und den eigenen Rechten und Pflichten zu Verunsicherung bei ausländischen Pflegekräften. Dies gilt besonders im Kontext von arbeitskulturellen Normen, da diese oft implizit sind und nicht ausgesprochen werden (Sakamoto et al. 2010, S. 148).

Die beschriebenen Barrieren verdeutlichen, dass geflüchtete Personen auf dem Arbeitsmarkt mit individuellen, strukturellen und institutionellen Barrieren konfrontiert sind. Strukturelle und institutionelle Barrieren können im Rahmen eines diversitätsorientierten Organisationsentwicklungsprozesses adressiert werden. Der Umgang mit individuell erlebten Barrieren bei der Arbeit im Krankenhaus könnte durch eine thematisch-fokussierte Vorbereitung der geflüchteten Personen gewinnbringend sein. So könnten im Rahmen eines Coachings sowohl interkulturelle als auch persönliche Anliegen im Kontext der Karriereentwicklung und der Arbeit in Gesundheitsberufen thematisiert werden.

\section{Interkulturelles Coaching}

In einer zunehmend globalisierten Welt steigt die Nachfrage nach interkulturellen Trainings oder Coachings (Schroll-Machl et al. 2018, S. 42). Interkulturelle Coachings werden in unterschiedlichen Kontexten angeboten und unterliegen daher auch keiner systematischen Definition (Nazarkiewicz und Krämer 2012, S. 7), wie das folgende Zitat zeigt: „Wenn von Coaching im interkulturellen Kontext die Rede ist, so kann das bedeuten: der Coachingpartner arbeitet für eine internationale Organisation, führt oder arbeitet mit Mitarbeitern aus verschiedenen Kulturen, das Coaching findet in einer Fremdsprache für den Coachee statt, der Inhalt ist interkultureller Natur oder es geht um eine Auslandsentsendung“ (Karboul 2009, S. 306).

$\mathrm{Zu}$ der Zielgruppe von interkulturellen Coachings können daher ausländische Fachkräfte, Mitarbeiter/innen, die ins Ausland gesandt werden, ausländische Studierende, aber auch alle Mitarbeitenden, die interkulturell sensibilisiert werden sollen, gehören. Für Barmeyer und Haupt (2007, S. 786) stellt das Interkulturelle Coaching eine Kombination aus klassischem Coaching und Interkulturalität dar. Dabei gehen sie davon aus, dass es notwendig ist, die Beziehung zwischen Coachingpartner/ innen und dem Kontext zu berücksichtigen und anliegen- und kontextspezifisch zu beraten. Nach Nazarkiewicz gilt Interkulturelles Coaching u.a. als „Form interkulturellen Lernens mit dem Effekt, dass bestimmte Ziele, Inhalte und Interventionen - wie bei einem Training - bereits vorgegeben sind oder sehr nahe gelegt werden“" (Nazarkiewicz und Krämer 2012, S. 70). Da es keine klare Abgrenzung zwischen einem Coaching und einem Training gibt, schlägt Schroll-Machl eine inhaltlichorientierte Richtung zur Unterscheidung vor: „Je mehr es um die Vermittlung von interkulturellem Know-how geht und die Inhalte deshalb zwangsläufig standardisierter und stereotyper sind, desto angemessener ist es, von interkulturellem Training zu sprechen (z. B. durchaus auch als Einzeltraining). Je mehr sich der Schwerpunkt auf die konkrete, fallbezogene Beratung verlagert, desto treffender ist der Lernprozess als interkulturelles Coaching zu bezeichnen (durchaus auch als Gruppencoaching). 
Dabei kann ein Training bereits Coaching-Elemente beinhalten, und ein Coaching braucht manche Trainingselemente" (Schroll-Machl et al. 2018, S. 42ff.).

Zentral für jede Art von (systemischem) Coaching ist es jedoch, ressourcenorientiert und lösungsorientiert zu arbeiten: „Im Coaching werden Potenziale des Ratsuchenden gefördert, damit er die vorhandenen Probleme und Aufgaben auf gesunde und stimmige Art lösen kann“ (Müller 2003, S. 53). Dabei muss eine Haltung des Nicht-Wissens eingenommen werden (Barthelmess 2016, S. 32), und Klient/ innen müssen als Expert/innen für ihre Entscheidungen betrachtet werden. „Coaching ist eine intensive und systematische Förderung ergebnisorientierter Problemund Selbstreflexionen sowie Beratung von Personen oder Gruppen zur Verbesserung der Erreichung selbstkongruenter Ziele oder zur bewussten Selbstveränderung und Selbstentwicklung“" (Greif 2008, S. 59).

Um ein Coaching-Angebot für geflüchtete Personen, die in Deutschland wieder in ihren erlernten Gesundheitsberufen arbeiten wollen, zu erstellen, bedarf es daher (1) einer systemischen Haltung, die in die Konzeptionierung des Angebots einfließt, und (2) der Berücksichtigung der genannten Barrieren, die geflüchtete Personen erleben. Das Ziel dieser Studie ist es, die Entwicklung und Durchführung des Coachings darzustellen und Ergebnisse der Evaluation kritisch zu diskutieren.

\section{Methoden}

\subsection{Entwicklung des Interkulturellen Coachings}

Das Interkulturelle Coaching war Teil eines Projekts zur Förderung von geflüchteten Personen in Gesundheitsberufen, das am Universitätsklinikum Hamburg-Eppendorf durchgeführt wurde. Das Projekt wurde vom Europäischen Sozialfonds finanziert und hatte das Ziel, geflüchtete Personen, die in ihren Herkunftsländern bereits in Gesundheitsberufen gearbeitet haben, bei ihrer beruflichen Integration zu unterstuitzen. Das Projekt bestand aus drei Maßnahmen: der Hospitation, dem Fachsprachenunterricht und dem Interkulturellen Coaching. Mithilfe des Interkulturellen Coachings sollten arbeits- und interkulturelle Aspekte der Arbeit im Gesundheitswesen in Deutschland adressiert werden. Die Ausgestaltung des Angebots konnte jedoch variieren, sodass anstelle eines Trainings mit vorgegebenen Ratschlägen die Form eines Coachings von den Autor/innen gewählt wurde.

Das interkulturelle Coaching verfolgte mehrere Ziele: 1) einen Raum zur Verfügung zu stellen, in dem die Teilnehmenden ihre Erfahrungen im Gesundheitswesen reflektieren und verarbeiten können, 2) Problem- und Selbstreflexionen unter den Teilnehmenden zu diversen inter- und arbeitskulturellen Aspekten der Arbeit zu ermöglichen, 3) Ressourcen der Teilnehmenden sichtbar zu machen und sie darin zu unterstützen, Strategien im Umgang mit herausfordernden Situationen zu entwickeln. Die Teilnahme am zwölfwöchigen Coaching war nicht verpflichtend, erfolgte aber gemeinsam mit anderen Teilnehmenden aus unterschiedlichen Gesundheitsberufen. Voraussetzung für die Teilnahme am Projekt war, dass die geflüchteten Personen in Hamburg gemeldet sind und den Anerkennungsprozess für ihre erlernten 


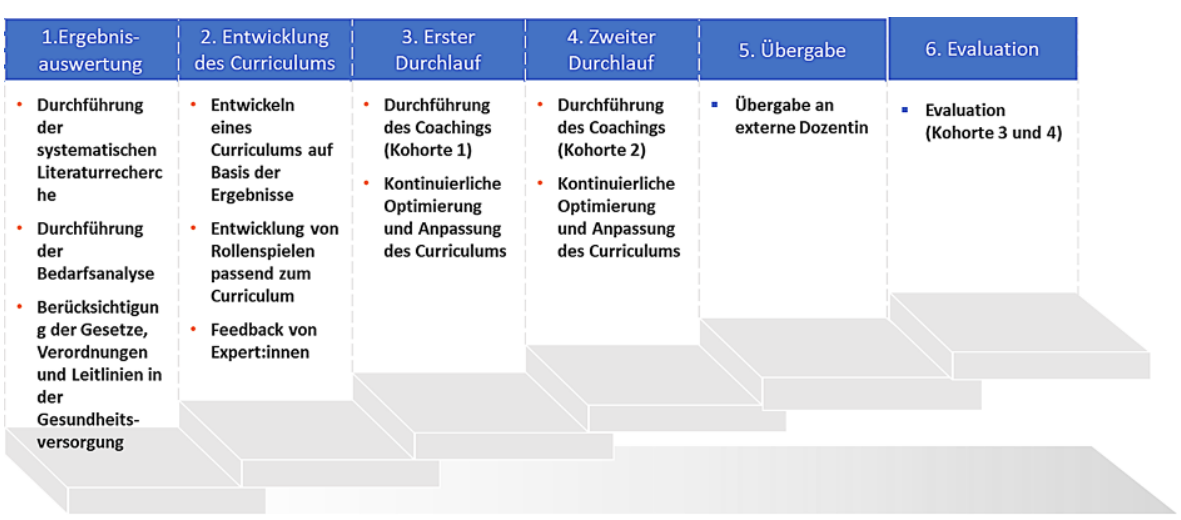

Abb. 1 Entwicklung des Interkulturellen Coachings

Gesundheits- und Heilberufe initiiert haben. Die Entwicklung des Interkulturellen Coachings erfolgte stufenweise (Abb. 1).

Um eine fundierte Basis für die Entwicklung der Inhalte zu haben, wurde eine systematische Literaturrecherche von internationalen Qualifikationsprogrammen für die berufliche Integration von geflüchteten und zugewanderten Fachkräften in Gesundheitsberufe durchgeführt. Dabei wurden zentrale Inhalte und Themen der Programme identifiziert (Khan-Gökkaya et al. 2019, S. 7 ff.). Die zweite Grundlage für die Entwicklung der Inhalte bildete eine bundesweite Befragung mit geflüchteten Fachkräften aus Gesundheitsberufen. Im Rahmen von Interviews wurden geflüchtete Fachkräfte und Betreuer/innen $(N=24)$ nach ihren Arbeitserfahrungen im Kontext der Gesundheitsberufe, besonders im Kontext von Arbeitskultur, und nach für sie relevanten Themen befragt. Darüber hinaus wurden Gesetze, Verordnungen und Leitlinien in der Gesundheitsversorgung berücksichtigt. Basierend auf den Vorarbeiten wurde ein Curriculum für ein zwölfwöchiges Interkulturelles Coaching entwickelt. Dieses Curriculum wurde mit einer Expert/innengruppe diskutiert und optimiert. Schwerpunkte der Coaching-Sitzungen waren:

- Das Gesundheitssystem in Deutschland,

- Rechte und Pflichten von Arbeitnehmer/innen und Arbeitgeber/innen,

- Kommunikation im Krankenhaus,

- Gesprächsführung mit Patient/innen,

- Schweigepflicht im Krankenhaus,

- Umgang mit Fehlern im Krankenhaus,

- Zusammenarbeit mit Vorgesetzten,

- Interprofessionelle Zusammenarbeit,

- Umgang mit Rassismus,

- Umgang mit Scham,

- Berufliche Integration in Deutschland (Bewerbungen).

Darüber hinaus wurde Zeit eingeräumt, um im Sinne eines Coachings aktuelle Anliegen oder vom Curriculum abweichende Aspekte der Teilnehmer/innen erörtern zu können. Die 2,5 h langen Coaching-Einheiten wurden mit einem kurzen Input 
der Trainerin zu den jeweiligen Themen eingeleitet und eröffneten den Raum für Erfahrungsaustausch und Diskussion in der Gruppe. Für einzelne Themen (Schweigepflicht, Umgang mit Fehlern, Interprofessionelle Zusammenarbeit, Umgang mit Rassismus und Umgang mit Scham) wurden spezifische Rollenspiele entwickelt. Diese wurden mit geschulten Simulationspatient/innen durchgeführt, um herausfordernde Situationen realitätsnah zu erproben.

Die Autor/innen führten das Interkulturelle Coaching modellhaft mit zwei Kohorten durch. Auf Basis der Rückmeldung der ersten beiden Kohorten wurde das Coaching optimiert. Anschließend wurden die Inhalte und Materialien des Coachings an eine externe, unabhängige Trainerin übergeben. Die Evaluation des Coachings erfolgte in den sich an die Übergabe anschließenden zwei Kohorten (Kohorte 3 und 4).

\subsection{Studiendesign ${ }^{1}$}

Das Coaching wurde mithilfe eines multi-methodalen Ansatzes evaluiert. Dabei wurden zwei $^{2}$ der vier Evaluationsstufen des Kirkpatrick Training Evaluation Modells (Zufriedenheit, Lernen, Verhalten, Ergebnisse) berücksichtigt. Für die Evaluation der ersten Stufe (Zufriedenheit) wurde das Coaching quantitativ geprüft. Da den Autor/ innen keine psychometrisch getesteten Instrumente für diesen spezifischen Kontext vorlagen, wurden Fragebögen entwickelt. Aufgrund der unterschiedlichen Sprachkenntnisse der Teilnehmenden wurde ein kurzer Fragebogen in leicht verständlicher Sprache entwickelt. Dieser Fragebogen wurde wöchentlich nach dem Interkulturellen Coaching verteilt und fragte mithilfe von drei Items Zufriedenheit, Relevanz der Themen und Verständnis der Inhalte mit einer 4-Punkte Likert-Skala ab. Zudem hatten die Teilnehmenden die Gelegenheit, offene Kommentare zu hinterlassen. Die Fragebögen wurden mithilfe von SPSS (Version 12) analysiert.

Der Schwerpunkt der Evaluation lag in der Durchführung und Auswertung qualitativer Interviews, um Erkenntnisse zur zweiten Evaluationsstufe (Lernen) generieren zu können. Ausgewählte Teilnehmende wurde mithilfe eines qualitativen Fallserienkonzepts (Yin 2014) zu positiven und negativen Erfahrungen, Optimierungsmöglichkeiten und Auswirkungen des Coachings befragt. Zu vier Messzeitpunkten (vor dem Start der Maßnahme, in der Mitte der Maßnahme, am Ende der Maßnahme und drei Monate nach dem Ende der Maßnahme) wurden Interviews mit 24 Teilnehmenden aus zwei unterschiedlichen Kohorten geführt. Dabei wurden die Teilnehmenden

\footnotetext{
1 Im Rahmen der Evaluation wurde das Gesamtprojekt (Fachsprachenunterricht, Coaching und Hospitation) evaluiert. Die Ergebnisse in diesem Artikel beziehen sich jedoch ausschließlich auf das Interkulturelle Coaching. Für die Evaluation des Gesamt-Projekts wurde neben den genannten Instrumenten ein mehrsprachiger Post-Fragebogen entwickelt, der nach Abschluss des Projekts an die Teilnehmenden verteilt wurde. In diesem Fragebogen wurden Verbesserungen der sprachlichen, professionellen und formalen Kompetenzen abgefragt. Da dieser Fragebogen sich nicht explizit auf das Interkulturelle Coaching, sondern auf das Gesamt-Projekt bezieht, wurde der Fragebogen in die Auswertung des Coachings nicht mit einbezogen.

2 Da die Teilnehmenden im Rahmen des Projektes nur eine Hospitation absolvierten und daher Tätigkeit nicht selbst ausführen durften, war es nicht möglich, Aussagen über die Auswirkungen auf das Verhalten zu treffen. Die qualitativen Ergebnisse liefern jedoch erste Hinweise, inwiefern das Coaching das Verhalten der Teilnehmenden beeinflusste.
} 
nach ihren Deutschkenntnissen und der Wahrscheinlichkeit ihrer Teilnahme für den gesamten Zeitraum ausgewählt. Die insgesamt $79^{3}$ Interviews wurden transkribiert und nach Mayring (2015) inhaltsanalytisch ausgewertet. Die Einwilligung für die Teilnahme an der Studie wurde im Vorfeld der Studie eingeholt und vor den Interviews mündlich besprochen.

\section{Ergebnisse}

\subsection{Beschreibung der Stichprobe}

Die Daten der Stichprobe sind in Tab. 1 zusammengefasst.

\subsection{Quantitative Ergebnisse}

Von den 29 Teilnehmenden der beiden Kohorten besuchten im Durchschnitt 18 Personen die einzelnen Sitzungen des interkulturellen Coachings, was einer durchschnittlichen Anwesenheit von 62,1\% entspricht. Dabei reichte die Anwesenheit von 10 Teilnehmenden (Abschlusssitzungen) bis zu 23 Teilnehmenden (Sitzungen zum Thema „Scham“). Zu den 24 Sitzungen liegen insgesamt 217 Feedbackbögen ${ }^{4}$ vor. Im Schnitt bewerteten die Teilnehmenden ihre Zufriedenheit mit dem interkulturellen Coaching mit ,sehr zufrieden“ (mean=3,58, SD=0,51, range=2-4). Außerdem wurden die Themen der einzelnen Sitzungen im Durschnitt als ,sehr wichtig“ (mean $=3,55, \mathrm{SD}=0,53$, range $=2-4$ ) bewertet.

Die höchste Zufriedenheit mit den Inhalten der Maßnahme (s. Tab. 2) und die am wichtigsten bewerteten Themen waren die Themen „Rassismus“ (M=3,89), „Bewerbungen“ (M=3,72) und „Schweigepflicht“ $(M=3,75)$ (s. Tab. 3). Den niedrigs-

Tab. 1 Beschreibung der Stichprobe

\begin{tabular}{lll}
\hline & Kohorte 3 & Kohorte 4 \\
\hline Stichprobengröße & $N=17$ & $N=12$ \\
Geschlecht & Männlich $(n=12)$ & Männlich $(n=6)$ \\
& Weiblich $(n=5)$ & Weiblich $(n=6)$ \\
Herkunftsland & Syrien $(n=12)$, & Syrien $(n=9)$ \\
& Algerien $(n=2)$, Kongo, Iran, Molda- & Afghanistan $(n=2)$ \\
Berufe & wien & Irak, Libyen \\
& Arzt/Ärztin $(n=14)$, & Arzt/Ärztin $(n=7)$ \\
& Psychologe $(n=2)$, & Physiotherapeut, \\
& Medizinisch-technischer Assistent & Zahnarzt, \\
& $(n=1)$ & Pharmazeut, \\
& & Medizinisch-technischer Assistent, \\
& & Pflegekraft \\
\hline
\end{tabular}

\footnotetext{
3 Insgesamt konnten 17 Interviews nicht geführt werden, da die Teilnehmenden entweder vorzeitig aus dem Projekt ausgestiegen sind oder für ein Follow-Up Interview nicht mehr erreicht werden konnten.

4 Die Ergebnisse beziehen sich auf die Anzahl der vorhandenen Feedbackbögen und beantworteten Items in der jeweiligen Sitzung.
} 
Tab. 2 Zufriedenheit mit dem Interkulturellen Coaching

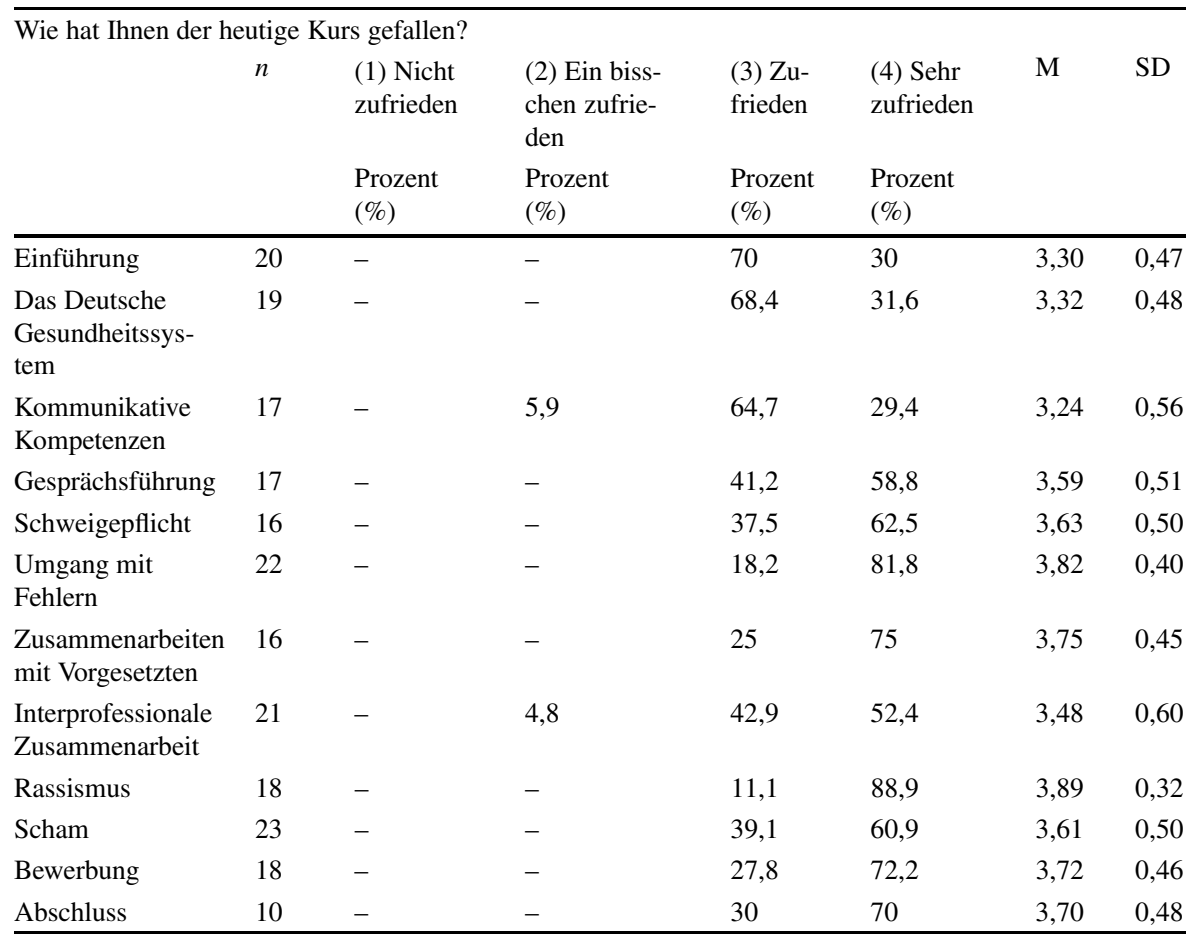

ten Zufriedenheitswert sowie die niedrigste Bewertung der Wichtigkeit erlangte das Thema „Kommunikative Kompetenzen“ $(M=3,24)$. Hier besteht möglicherweise ein Zusammenhang dazu, wieviel Prozent der Sitzungen die Teilnehmenden verstanden haben. Mit nur $53 \%$ der Teilnehmenden, die angaben, bei dem Thema „Kommunikative Kompetenzen“ 75-100\% verstanden zu haben $(m=3,47)$, liegt dieser Kurs das sprachliche Verständnis betreffend auf dem letzten Platz. Generell war die Verständlichkeit der Inhalte (Tab. 4) unter den Teilnehmenden dennoch hoch, und im Schnitt gaben diese an, $75 \%$ der Inhalte zu verstehen.

\subsection{Qualitative Ergebnisse}

Die qualitative Analyse führte zu der Bildung von drei Kategorien: (1) positive Aspekte im Hinblick auf das Coaching, (2) negative Aspekte im Hinblick auf das Coaching sowie (3) Lernzuwachs und Verbesserung von Kompetenzen durch das Coaching. Da im Rahmen dieser Studie keine Zeiteffekte beobachtet werden konnte, werden die Ergebnisse im Folgenden entlang der Kategorien dargestellt und nicht entlang der Messzeitpunkte. Dabei muss berücksichtigt werden, dass es Überschneidungen zwischen der ersten (positive Aspekte) und dritten Kategorie (Lernzuwachs) gibt, da diese nicht immer voneinander getrennt werden können. Entsprechend werden im Folgenden zunächst Ergebnisse der ersten und dritten Kategorie dargestellt und abschließend auf negative Aspekte eingegangen. 
Tab. 3 Relevanz der Themen im Interkulturellen Coaching

\begin{tabular}{|c|c|c|c|c|c|c|c|}
\hline \multicolumn{8}{|c|}{ Wie wichtig war das heutige Thema für Sie? } \\
\hline & \multirow[t]{2}{*}{$n$} & $\begin{array}{l}\text { (1) Nicht } \\
\text { wichtig }\end{array}$ & \multirow{2}{*}{$\begin{array}{l}\text { (2) Ein biss- } \\
\text { chen wichtig } \\
\text { Prozent } \\
(\%)\end{array}$} & \multirow{2}{*}{$\begin{array}{l}\text { (3) Wich- } \\
\text { tig } \\
\text { Prozent } \\
(\%)\end{array}$} & \multirow{2}{*}{$\begin{array}{l}\text { (4) Sehr } \\
\text { wichtig } \\
\text { Prozent } \\
(\%)\end{array}$} & \multirow[t]{2}{*}{ M } & \multirow[t]{2}{*}{ SD } \\
\hline & & $\begin{array}{l}\text { Prozent } \\
(\%)\end{array}$ & & & & & \\
\hline Einführung & 19 & - & 5,3 & 63,2 & 31,6 & 3,26 & 0,56 \\
\hline $\begin{array}{l}\text { Das Deutsche Ge- } \\
\text { sundheitssystem }\end{array}$ & 18 & - & - & 55,6 & 44,4 & 3,44 & 0,51 \\
\hline $\begin{array}{l}\text { Kommunikative } \\
\text { Kompetenzen }\end{array}$ & 17 & - & 5,9 & 76,5 & 17,6 & 3,12 & 0,49 \\
\hline Gesprächsführung & 17 & - & - & 29,4 & 70,6 & 3,71 & 0,47 \\
\hline Schweigepflicht & 16 & - & - & 25 & 75 & 3,75 & 0,45 \\
\hline $\begin{array}{l}\text { Umgang mit } \\
\text { Fehlern }\end{array}$ & 22 & - & - & 31,8 & 68,2 & 3,68 & 0,48 \\
\hline $\begin{array}{l}\text { Zusammenarbeit } \\
\text { mit Vorgesetzten }\end{array}$ & 16 & - & - & 43,8 & 56,3 & 3,56 & 0,51 \\
\hline $\begin{array}{l}\text { Interprofessionale } \\
\text { Zusammenarbeit }\end{array}$ & 21 & - & 4,8 & 47,6 & 47,6 & 3,43 & 0,60 \\
\hline Rassismus & 18 & - & - & 22,2 & 77,8 & 3,78 & 0,43 \\
\hline Scham & 23 & - & - & 47,8 & 52,2 & 3,52 & 0,51 \\
\hline Bewerbung & 18 & - & - & 22,2 & 77,8 & 3,78 & 0,43 \\
\hline Abschluss & 10 & - & - & 40 & 60 & 3,60 & 0,52 \\
\hline
\end{tabular}

Tab. 4 Verständnis

\begin{tabular}{|c|c|c|c|c|c|c|c|}
\hline \multicolumn{8}{|c|}{ Wie viel Prozent von dem Kurs heute haben Sie verstanden? } \\
\hline & \multirow[t]{3}{*}{$n$} & & & & & \multirow[t]{3}{*}{ M } & \multirow[t]{3}{*}{ SD } \\
\hline & & $0-25 \%$ & $25-50 \%$ & $50-75 \%$ & $75-100 \%$ & & \\
\hline & & $\begin{array}{l}\text { Prozent } \\
(\%)\end{array}$ & $\begin{array}{l}\text { Prozent } \\
(\%)\end{array}$ & $\begin{array}{l}\text { Prozent } \\
(\%)\end{array}$ & $\begin{array}{l}\text { Prozent } \\
(\%)\end{array}$ & & \\
\hline Einführung & 20 & - & - & 20 & 80 & 3,80 & 0,41 \\
\hline $\begin{array}{l}\text { Das Deutsche Ge- } \\
\text { sundheitssystem }\end{array}$ & 19 & - & 5,3 & 31,6 & 63,2 & 3,58 & 0,61 \\
\hline $\begin{array}{l}\text { Kommunikative } \\
\text { Kompetenzen }\end{array}$ & 17 & - & 5,9 & 41,2 & 52,9 & 3,47 & 0,62 \\
\hline Gesprächsführung & 16 & - & 6,3 & 31,3 & 62,5 & 3,56 & 0,63 \\
\hline Schweigepflicht & 14 & - & - & 21,4 & 78,6 & 3,79 & 0,43 \\
\hline $\begin{array}{l}\text { Umgang mit Feh- } \\
\text { lern }\end{array}$ & 22 & - & - & 31,8 & 68,2 & 3,68 & 0,48 \\
\hline $\begin{array}{l}\text { Zusammenarbeit } \\
\text { mit Vorgesetzten }\end{array}$ & 14 & - & 7,1 & 35,7 & 57,1 & 3,50 & 0,65 \\
\hline $\begin{array}{l}\text { Interprofessionale } \\
\text { Zusammenarbeit }\end{array}$ & 18 & - & - & 27,8 & 72,2 & 3,72 & 0,46 \\
\hline Rassismus & 18 & - & - & 33,3 & 66,7 & 3,67 & 0,49 \\
\hline Scham & 22 & - & - & 31,8 & 68,2 & 3,68 & 0,48 \\
\hline Bewerbung & 17 & - & - & 17,6 & 82,4 & 3,82 & 0,39 \\
\hline Abschluss & 10 & - & - & 10 & 90 & 3,90 & 0,32 \\
\hline
\end{tabular}


Die Teilnehmenden waren generell zufrieden mit dem Coaching. Sie gaben an, dass die Themen relevant waren und sie nicht nur über das Gesundheitssystem, sondern auch über kulturelle Aspekte des Zusammenlebens in Deutschland etwas lernen konnten:

Ich bin seit zwei Jahren in Deutschland und ich hatte keinen richtigen Kontakt zu der deutschen Kultur. Und zu wissen, was hier ein Tabu ist, was nicht richtig ist, was man nicht machen soll. Das ist gut zu wissen, bevor man Kontakt zu Patienten hat. (Ärztin aus Syrien)

Außerdem empfanden die Teilnehmenden das Coaching nicht nur hilfreich für ihre berufliche Situation, sondern auch für das Leben in Deutschland:

Es [das Coaching] hat mit dem Alltagsleben zu tun. [...] Sie [die Trainerin] hat ein schweres Thema mit uns diskutiert - Rassismus. Etwas, was wir jeden Tag erleben. [Wir sprachen darüber], wie kann man eine Lösung finden in dieser Situation oder einen Weg raus aus der Situation. [...] Nicht nur medizinisch, sondern allgemein. (Arzt aus Libyen)

Die Teilnehmenden berichteten auch, dass sie durch das Coaching unterschiedliche Meinungen vergleichen konnten und die Rollenspiele diese Meinungen erfahrbar machten:

Durch die Themen, durch die Diskussion darüber, und mit Kollegen auch interkulturell. Jeder hat vor einem Land und hat eine eigene Kultur. Und wir haben auch -. Wir haben das verglichen und mit Rollenspiel auch etwas, ein bisschen lebendig gesehen haben. (Arzt aus Syrien)

Weiterhin wurden sie durch das Coaching für die Arbeit in kulturell und sprachlich diversen Teams vorbereitet:

I: Glauben Sie, das interkulturelle Coaching hilft Ihnen?

P: Ich glaube, wir werden in Deutschland mit verschiedene Leute arbeiten. Deshalb müssen wir das jetzt auch im Team - man soll bereit sein, mit verschiedene Leute, die verschiedene Hintergrund haben, immer arbeiten. Weil in Krankenhäuser habe ich bemerkt, dass unterschiedliche Leute, nicht nur Deutsche oder, aus verschiedene Länder. Ja, es hilft. (Arzt aus Syrien)

Dabei half es ihnen besonders, dass sie im Coaching ihre Meinung äußern konnten:

Ja, ich kann [...] meine Meinung äußern und die anderen akzeptieren, einfach akzeptieren. Nicht -, sage ich nicht, ich bin richtig und die anderen sind falsch. Das ist wichtig auch, dass wir die anderen akzeptieren, die andere Meinungen.

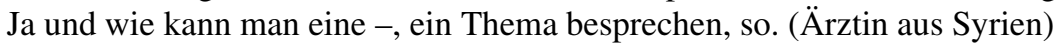
Also, wenn wir über ein Thema gesprochen haben, wir konnten über dieses Thema frei uns äußern. Und was sind unsere Meinungen. Also ist das kulturell in Ordnung, bin ich in Ordnung? Wir konnten alles das frei sagen, ohne das Gefühl, dass ist nicht akzeptabel. (Ärztin aus Syrien) 
Außerdem beschrieben sie, dass der Austausch in der Gruppe hilfreich war:

Und die Meinung meiner Kollegen kennenzulernen. Das ist für mich sehr interessant. [...] Wir haben über Rassismus geredet und es war sehr interessant. Weil manchmal, dieses bestimmte Thema ist schwierig, mit anderen deutschen Leuten zu reden, weil sie diese Situation nicht erlebt haben. Sie können sich dazu nicht relaten. (Arzt aus Libyen)

Zudem wurden sie in dem Umgang mit herausfordernden Situationen gestärkt:

Wenn wir über ein Thema diskutieren, können wir mehr darüber nachdenken. Zum Beispiel das Thema „Fehler“. Wenn ein Fehler passiert, wie kann man damit umgehen, was kann ich tun? Ich hatte keine Idee dazu. Aber dann, als ich im Unterricht gehört habe [dachte ich] ,,das ist etwas, was ich machen kann, wenn Fehler passieren“. (Ärztin aus Syrien)

Die Teilnehmenden betonten, dass die Rollenspiele in diesem Kontext besonders hilfreich waren, da sie alltagsnahe Situationen einüben konnten:

Einmal diese mit Schauspielen. Einmal sollte ich eine Situation -, das war aber ein Fehler. Ich habe einen Fehler mit Patient -, ich habe einen Patient vergessen, ihm Medikamente zu geben oder etwas so [bezieht sich auf das Szenario aus dem Rollenspiel]. Und wie kann ich damit umgehen? Und ich habe das gemacht mit einer Schauspielerin und wo die diese Feedback war gut. Was war gut? Was war nicht gut? Das ist eine sehr gute Methode oder so, damit man lernen, wie kann man mit diese Situationen umgehen oder machen (Ärztin aus Syrien)

Es hat uns sehr geholfen. Ich und meine Kollegen haben über die Rollenspiele gesprochen und man hat nicht den Stress danach und kann viele Sachen aufklären und [wissen was] kann man fragen. Deshalb der Kurs hat mir sehr geholfen. (Arzt aus Syrien)

Darüber hinaus gab es auch kritische Kommentare. So wurde angemerkt, dass die Themen sich mit Themen aus zuvor besuchten Sprachkursen für Mediziner/innen deckten:

Aber die Coaching. Ja, gibt es eigene Thema für die Shame und Rassismus. Und die Gesundheitssystem. Aber ich glaube, jeder Teilnehmer hat schon diese Thema in die medizinische Sprachkurs gehabt (Arzt aus Syrien).

Ein weiterer Kritikpunkt bezog sich auf die Relevanz der Themen:

Zum Beispiel heute war über die Konflikte oder so. Also wir haben schon gearbeitet. Ich glaube, wir sind nicht neu mit die Konflikte und das ist nicht unterschiedlich zwischen Syrien oder hier. Man muss mit dem Chef oder mit den Kollegen, wir sind ein Team. Ich finde, es gibt nichts neu. Wenn eine Person mich ignorieren, ich muss mit ihm über die Probleme besprechen. Ich finde das nicht neu. Für mich. Vielleicht ist das neu für die anderen, aber für mich ist das nicht neu. (Ärztin aus Syrien).

Einzelne Teilnehmende beschrieben einen inneren Konflikt mit der Uhrzeit des Interkulturellen Coachings, da dieser Freitagnachmittag stattfand. Einige Teilneh- 
menden fühlten sich mit dieser Uhrzeit unwohl, da das Freitagsgebet für Muslime ebenfalls freitags nachmittags stattfindet:

Nur leider am Freitag, aber das ist kein großes Problem -, ich kann freitags nicht in die Moschee gehen. Ich kann das nicht machen. [...] Das ist sehr wichtig für mich, das ist meine Religion. Und das macht mich glücklich. (Medizinischtechnischer Assistent aus Syrien)

\section{Diskussion}

Ziel dieser Studie war es, die Entwicklung und Evaluation einer Interkulturellen Coachingmaßnahme zur besseren beruflichen Integration von geflüchteten Fachkräften in Gesundheitsberufen darzulegen. Aufgrund der spezifischen Zielgruppe und dem spezifischen Arbeitskontext wurde ein Coaching-Konzept entwickelt, das auf die Zielgruppe und auf relevante interkulturelle Aspekte der Arbeit im Gesundheitswesen zugeschnitten ist. Didaktisch bestand die Maßnahme aus Elementen des Trainings und des Coachings. Durch die Vorgabe bestimmter Themen wurde die systemische Haltung des Nicht-Wissens jedoch ein Stück weit verlassen. Auch die Teilnehmenden bezogen sich auf das Coaching im Kontext eines Kurses oder Unterrichts. Zudem wird die Intimität und Neutralität zwischen den Coachpartner/innen eingeschränkt, da eine Gruppensituation die Dynamik verändert. Gleichwohl deuten die Ergebnisse an, dass während der Sitzungen eine offene Atmosphäre hergestellt werden konnte, in der unterschiedliche Meinungen gewertschätzt und nicht auf ihre „Richtigkeit“ hin bewertet wurden.

Die Ergebnisse verdeutlichen auch, dass die behandelten Aspekte eine hohe Relevanz für die Teilnehmenden hatten. Da arbeitskulturelle Normen häufig implizit sind, kann es sinnvoll sein, in den Austausch über diese zu gehen und von den Erfahrungen anderer zu lernen. Gleichzeitig ist es wichtig, zu berücksichtigen, dass Arbeitskultur im Kontext des Gesundheitswesens sich auf verschiedene Konzepte beziehen kann. Dabei kann es sich um Leitlinien, Normen oder Standards handeln, die vorgegeben sind oder sich durch eine Routine etabliert haben (Klingler et al. 2018, S. 17). Zudem sind Unterschiede in der Arbeitskultur nicht zwangsläufig mit unterschiedlichen kulturellen Wertesystemen in Verbindung zu bringen, sondern mit einer fachlichen und betrieblichen Sozialisation, die sich an den nationalen Gesundheits- und Sozialsystemen orientiert (Pütz et al. 2019, S. 173).

Um diese inter- und arbeitskulturellen Gemeinsamkeiten und Unterschiede zu reflektieren, war es für die Ziele des Coachings sinnvoll, dass es ein Gruppencoaching war, da die Gruppe aus mehreren Teilnehmer/innen bestand, die ähnliche Erfahrungen in ähnlichen Settings machten. Obwohl diese Form des Gruppencoachings deutlich von klassischen Coachingformaten abweicht, wurde durch die qualitativen Ergebnisse deutlich, dass das gemeinsame Teilen einer (oder mehrerer) Erfahrungen, z.B. rassistischer Diskriminierung, wichtig für die Teilnehmenden war. Das Feedback der Gruppe wurde auch im Hinblick auf die Rollenspiele als nützlich erachtet. Die Teilnehmer/innen hatten dadurch die Möglichkeit, in einem geschützten Rahmen herausfordernde Situationen nachzustellen und mit den ihnen zur Verfügung stehen- 
den Sprachkenntnissen die dargestellten Situationen zu bewältigen und ihre eigenen Strategien zu finden. Negative Effekte durch die Gruppendynamik wurden im Kontext des Interkulturellen Coachings nicht benannt ${ }^{5}$. Es kann jedoch angenommen werden, dass die unterschiedlichen Sprachniveaus und Berufe der Teilnehmenden einen Einfluss auf das Befinden der Teilnehmenden hatten.

Die Sitzungen zu den Themen „Rassismus“ und „Bewerbungen“ wurden als am wichtigsten bewertet und führten zu einer hohen Zufriedenheit bei den Teilnehmenden. Eine Erklärung für die Relevanz beider Themen könnte darin liegen, dass sie als größte Hindernisse auf dem Weg zur Partizipation am Arbeitsmarkt gesehen werden. Bewerbungstrainings und Vorbereitungen auf Bewerbungsgespräche sind oftmals Teil von Integrationsprogrammen und Trainingsangeboten für geflüchtete Personen (Konle-Seidl und Bolits 2016, S. 41), da Sprachbarrieren und eine mangelnde Vertrautheit mit den Bewerbungsprozessen und -abläufen das Erstellen von passenden Bewerbungsunterlagen erschweren können. Entsprechend scheint es nicht verwunderlich, dass die Sitzung zum Thema „Bewerbungen“ als wichtig bewertet wurde. Auch die Zufriedenheit mit dieser Sitzung könnte dadurch erklärt werden, dass praktikable Tipps zum Bewerbungsverfahren besprochen wurden. Die Relevanz des Themas „Rassismus“ kann ebenfalls damit erklärt werden, dass geflüchtete Personen sowohl im Arbeitskontext als auch in anderen Lebensbereichen rassistische Diskriminierung erfahren. Eine Studie der Antidiskriminierungsstelle kommt zu dem Ergebnis, das geflüchtete Personen auf dem Wohnungsmarkt, auf dem Arbeitsmarkt sowie bei dem Zugang zu Waren und Dienstleistungen am häufigsten Diskriminierung erleben (Antidiskriminierungsstelle des Bundes 2016, S. 8). Die Studie kommt ebenfalls zu dem Ergebnis, dass kaum Wissen über Diskriminierungsschutz und Handlungsmöglichkeiten vorhanden ist (S. 26). Es ist daher möglich, dass die Teilnehmenden aus der Sitzung zum Thema „Rassismus“ und aus dem Rollenspiel nützliches Wissen und Strategien zum Umgang mit Diskriminierung mitnehmen konnten und sie daher auch zufriedener mit dieser Sitzung waren.

Die qualitativen Ergebnisse verdeutlichen aber auch, dass nicht alle Themen als relevant betrachtet wurden. Interessant ist hier vor allem die Aussage einer Teilnehmerin, dass z. B. das Thema „Umgang mit Kolleg/innen und Vorgesetzten“ keinen neuen Aspekt für sie beinhaltete und sich nicht von ihren Erfahrungen in Syrien unterschied. Hier wird deutlich, dass von den Autor/innen angenommen wurde, dass es Unterschiede z. B. in der interprofessionellen Zusammenarbeit oder in der Hierarchie geben könnte, die adressiert werden müssten, obwohl diese für die Teilnehmerin kein Hindernis darstellten. Dieses Beispiel veranschaulicht, dass (angenommene) arbeits-, und interkulturelle Aspekte nur von den Klient/innen auf ihre Relevanz hin beurteilt werden können, da sie die Expert/innen für sich und die für sie relevanten Themen sind. Zum anderen wurde kritisiert, dass die Themen eine Wiederholung

\footnotetext{
5 Viele Teilnehmer/innen kritisierten im Kontext des Deutsch-Unterrichts die Zusammensetzung der Gruppe, da alle Teilnehmer/innen unterschiedliche Sprachniveaus hatten und unterschiedliche Berufe ausübten. Die meisten Teilnehmer/innen waren jedoch Ärzt/innen. Angehörige auch nicht-ärztlicher Berufe fühlten sich im Deutsch-Unterricht nicht ausreichend berücksichtigt, da die Themen zu sehr auf ärztliche Themen abzielten. Es ist anzunehmen, dass sich dies auch auf das Interkulturelle Coaching bezieht, aber öfter im Kontext des Deutsch-Unterrichts angesprochen wurde, da es dort aufgrund der Fachbegriffe sichtbarer ist.
} 
darstellten oder zu weit gefasst waren. Hier ist es besonders wichtig, den Teilnehmenden auch die Möglichkeit zu geben, eigene Themen einzubringen.

Ein weiterer Kritikpunkt bezog sich auf die Uhrzeit des Coachings, das mit der Uhrzeit des Freitagsgebets kollidiert. Da die Teilnahme am Freitagsgebet für die Teilnehmenden zur Stressbewältigung diente und zu ihrem Wohlbefinden beitrug, fühlten sie sich hin- und hergerissen zwischen der Teilnahme am Coaching und am Freitagsgebet. Religion kann eine Bewältigungsstrategie für geflüchtete Personen sein (Brune et al. 2002, S. 456) und eine Quelle emotionaler und kognitiver Unterstützung darstellen (Gozdziak und Shandy 2002, S. 129). Hier wird deutlich, wie wichtig die Rolle von Unterstützungssystemen sein kann und dass es notwendig ist, diese Unterstützungssysteme bei der Entwicklung von Angeboten mit zu berücksichtigen.

Insgesamt deuten die Ergebnisse darauf hin, dass das Coaching für die Teilnehmenden zielführend war. Dennoch stellt sich in diesem Zusammenhang die Frage der Nachhaltigkeit. Vor dem Hintergrund der Barrieren, die geflüchtete Personen auf dem Arbeitsmarkt erleben, kann es im Rahmen von Unterstützungsangeboten, z. B. von Coachings, wichtig sein, geflüchtete Personen auf die Arbeit vorzubereiten, ihre Ressourcen sichtbar zu machen und sie in ihrer Handlungsfähigkeit zu stärken. Darüber hinaus ist es zur erfolgreichen und nachhaltigen Partizipation am Arbeitsmarkt notwendig, strukturelle und institutionelle Barrieren zu adressieren. Dazu zählt u.a., dass Benachteiligungen und Diskriminierungen innerhalb der Organisationen entgegengewirkt werden muss, um die Zufriedenheit am Arbeitsplatz zu erhöhen und eine respektvolle und wertschätzende Atmosphäre zu erzeugen (Kehoe et al. 2016, S. 1019). Dies gilt auch für das Coaching selbst und damit für die Rolle des/r Coach/in. Für die Rolle der Coaches in dem hier beschriebenen Coaching war es zum einen notwendig, eine ,kulturreflexive“ (Nazarkiewicz 2018, S. 22) Haltung zu entwickeln, um Verletzungen bei den Teilnehmenden vorzubeugen. Kulturreflexiv bezieht sich dabei auf ein dynamisches Verständnis von Kultur, das nicht eine homogene Einheitskultur basierend auf Landesgrenzen beschreibt (ebd., S. 22), sondern sich in unterschiedlichen Facetten, Identitäts- und Diversitätsdimensionen wiederfindet. Ein bewusster und kritischer Umgang mit dem Begriff Kultur ist daher enorm wichtig, um der Gefahr einer Kulturalisierung vorzubeugen (ebd., S. 28). Weiterhin ist es wichtig als Coach/in, eine diversitätskompetente Haltung zu entwickeln und „(unbewusste) Exklusionsmechanismen aufzudecken und deren Reinszenierung als Berater/in zu vermeiden, um so auch neuerlichen Verletzungen auf Klient/innenseite vorzubeugen“ (Abdul-Hussain und Baig 2009, S. 57). Konkret bedeutet das, dass der/die Coach/in sich erstens Wissen um gesellschaftliche Konstruktionen von Ungleichverhältnissen aneignen muss, zweitens Diskriminierungsstrukturen und die Rolle der eigenen Werte und Einstellungen dabei erkennen muss und auf Basis dessen drittens die eigenen Positionierungen und Privilegien reflektieren muss (ebd., S. 136), um produktiv mit dem Wissen umzugehen, persönliche und professionelle Solidarität zu entwickeln und Empowermentprozesse zu unterstützen (ebd., S. 57). 


\section{Limitationen und Stärken}

Bei der Interpretation der Ergebnisse müssen methodische Limitationen berücksichtigt werden. Da die Teilnehmenden des Coachings unterschiedliche Sprachkenntnisse hatten, mussten Studieninstrumente gewählt werden, die für alle Teilnehmenden verständlich waren. Die Fragebögen wurden daher selbst entwickelt und aufgrund einer kleinen Stichprobenzahl nicht psychometrisch getestet. Daher lassen sich auch keine Aussagen über Subgruppen treffen. Auch bei der Durchführung der qualitativen Studien wurden nur Teilnehmende ausgewählt, die ausreichend Deutsch sprechen konnten, um das Interview zu führen. Sprachbarrieren sowie sozial erwünschte Antworten können daher die Ergebnisse der Interviews verzerren. Weiterhin konnten keine trennscharfen Kategorien im Rahmen der Inhaltsanalyse gebildet werden, da die Evaluation des Coachings im Rahmen des Gesamtprojektes erfolgte. Darüber hinaus können auf Basis des Forschungsdesigns die Ergebnisse nicht generalisiert werden. Die Wahrscheinlichkeit für eine Erinnerungsverzerrung (recall bias) wurde allerdings minimiert, indem Interviews zu mehreren Zeitpunkten geführt wurden. Durch eine multimethodale Vorgehensweise wurde ermöglicht, dass alle Teilnehmenden in die Evaluation eingeschlossen wurden und Aussagen zur Prozess- und Ergebnisqualität des Coachings getroffen werden konnten.

Funding Open Access funding provided by Projekt DEAL.

Open Access Dieser Artikel wird unter der Creative Commons Namensnennung 4.0 International Lizenz veröffentlicht, welche die Nutzung, Vervielfältigung, Bearbeitung, Verbreitung und Wiedergabe in jeglichem Medium und Format erlaubt, sofern Sie den/die ursprünglichen Autor(en) und die Quelle ordnungsgemäß nennen, einen Link zur Creative Commons Lizenz beifügen und angeben, ob Änderungen vorgenommen wurden.

Die in diesem Artikel enthaltenen Bilder und sonstiges Drittmaterial unterliegen ebenfalls der genannten Creative Commons Lizenz, sofern sich aus der Abbildungslegende nichts anderes ergibt. Sofern das betreffende Material nicht unter der genannten Creative Commons Lizenz steht und die betreffende Handlung nicht nach gesetzlichen Vorschriften erlaubt ist, ist für die oben aufgeführten Weiterverwendungen des Materials die Einwilligung des jeweiligen Rechteinhabers einzuholen.

Weitere Details zur Lizenz entnehmen Sie bitte der Lizenzinformation auf http://creativecommons.org/ licenses/by/4.0/deed.de.

\section{Literatur}

Abdul-Hussain, S., \& Baig, S. (2009). Diversity in Supervision, Coaching und Beratung. Wien: facultas.

Aden, S., Schmitt, C., Uçan, Y., Wagner, C., \& Wienforth, J. (2019). Partizipative Fluchtmigrationsforschung. Eine Suchbewegung. Zeitschrift für Flucht- und Flüchtlingsforschung, 3(2), 302-319. https:// doi.org/10.5771/2509-9485-2019-2-302.

Antidiskriminierungsstelle des Bundes (2016). Diskriminierungsrisiken für Geflüchtete in Deutschland. Eine Bestandsaufnahme der Antidiskriminierungsstelle des Bundes. https://www.antidiskriminierungs stelle.de/SharedDocs/Downloads/DE/publikationen/Expertisen/Diskriminierungsrisiken_fuer_ Gefluechtete_in_Deutschland.pdf?_blob=publicationFile\&v=4. Zugegriffen: 7. Apr. 2020.

Barmeyer, C. I., \& Haupt, U. (2007). Interkulturelles Coaching. In J. Straub, A. Weidemann \& D. Weidemann (Hrsg.), Handbuch Interkulturelle Kommunikation und Kompetenz (S. 784-793). Stuttgart: Metzler. 
Barthelmess, M. (2016). Die systemische Haltung. Was systemisches Arbeiten im Kern ausmacht. Göttingen: Vandenhoeck \& Ruprecht.

Brune, M., Haasen, C., Krausz, M., Yagdiran, O., Bustos, E., \& Eisenman, D. (2002). Belief systems as coping factors for traumatized refugees: a pilot study. European Psychiatry, 17(8), 451-458.

Bundesamt für Migration und Flüchtlinge (2011). Anerkennung und Berufszugang für Ärzte und Fachärzte mit ausländischen Qualifikationen in Deutschland. Informationsbroschüre für Zugewanderte und Beratungsstellen. https://www.bda.de/files/Broschueren/BAMF_Informationsbroschuere_Aerzte_ web.pdf. Zugegriffen: 7. Apr. 2020.

Bundesamt für Migration und Flüchtlinge (2020). Aktuelle Zahlen. Ausgabe Januar 2020. https:// www.bamf.de/SharedDocs/Anlagen/DE/Statistik/AsylinZahlen/aktuelle-zahlen-januar-2020.pdf? blob=publicationFile\&v=3. Zugegriffen: 7. Apr. 2020.

Cohn, S., Alenya, J., Murray, K., Bhugra, D., De Guzman, J., \& Schmidt, U. (2006). Experiences and expectations of refugee doctors: Qualitative study. British Journal of Psychiatry, 189, 74-78. https:// doi.org/10.1192/bjp.bp.105.010975.

Colic-Peisker, V., \& Tilbury, F. (2003). "Active" and "passive" resettlement: the influence of support services and refugees' own resources on resettlement style. International Migration, 41, 61-91.

Desiderio, M. V. (2016). Integrating refugees into host country labor markets: challenges and policy options. Washington: Migration Policy Institute.

Diekmann, D., \& Fereidooni, K. (2019). Diskriminierungs- und Rassismuserfahrungen geflüchteter Menschen in Deutschland: Ein Forschungsüberblick. Zeitschrift für Flucht- und Flüchtlingsforschung, 3(2), 343-360. https://doi.org/10.5771/2509-9485-2019-2-343.

Etzold, B. (2017). Arbeit trotz Asyl? Erlebte Chancen und Hürden von Geflüchteten beim Zugang zu Arbeit. In M. Becker, V. Kronenberg \& H. Pompe (Hrsg.), Fluchtpunkt Integration: Panorama eines Problemfeldes (S. 319-353). Wiesbaden: VS.

Gozdziak, E. M., \& Shandy, D. J. (2002). Editorial introduction: religion and spirituality in forced migration special issue: religion and forced migration: editorial introduction. Journal of Refugee Studies, 2 , $129-135$.

Greif, S. (2008). Coaching und ergebnisorientierte Selbstreflexion. Göttingen: Hogrefe.

Jirovsky, E., Hoffmann, K., Maier, M., \& Kutalek, R. (2015). "Why should I have come here?" A qualitative investigation of migration reasons and experiences of health workers from sub-Saharan Africa in Austria. BMC Health Services Research, 15, 74. https://doi.org/10.1186/s12913-015-0737-z.

Johansson, S. (2016). Die ungleiche Teilhabechancen für geflüchtete Menschen in Deutschland: Rahmenbedingungen der Lebenssituation von Flüchtlingen. In G. G. Goth \& E. Severing (Hrsg.), Asylsuchende und Flüchtlinge in Deutschland: Erfassung und Entwicklung von Qualifikationen für die Arbeitsmarktintegration (S. 19-38). Bielefeld: wbv.

Karboul, A. (2009). Coaching im internationalen Kontext. Organisationsberatung Supervision Coaching, 16(3), 305-319.

Kehoe, A., McLachlan, J., Metcalf, J., Forrest, S., Carter, M., \& Illing, J. (2016). Supporting international medical graduates' transition to their host-country: realist synthesis. Medical Education, 50(10), 1015-1032. https://doi.org/10.1111/medu.13071.

Khan-Gökkaya, S., Higgen, S., \& Mösko, M. (2019). Qualification programmes for immigrant health professionals: A systematic review. PLOS ONE, 14(11), e224933. https://doi.org/10.1371/journal.pone. 0224933.

Klingler, C., \& Marckmann, G. (2016). Difficulties experienced by migrant physicians working in German hospitals: a qualitative interview study. Human Resources for Health, 14(1), 57. https://doi.org/10. 1186/s12960-016-0153-4.

Klingler, C., Ismail, F., Marckmann, G., \& Kuehlmeyer, K. (2018). Medical professionalism of foreignborn and foreign-trained physicians under close scrutiny: A qualitative study with stakeholders in Germany. PLoS ONE, 13(2), 2018.

Konle-Seidl, R., \& Bolits, G. (2016). Labour market integration of refugees: Strategies and good practices. European Parliament: Policy Department A: Economic and Scientific Policy, Brussels. https:// www.europarl.europa.eu/RegData/etudes/STUD/2016/578956/IPOL_STU(2016)578956_EN.pdf. Zugegriffen: 7. Apr. 2020.

Mayring, P. (2015). Qualitative Inhaltsanalyse. Grundlagen und Techniken. Weinheim, Basel: Beltz.

Müller, G. (2003). Systemisches Coaching im Management. Das Praxisbuch für Neueinsteiger und Profis. Weinheim: Beltz.

Nazarkiewicz, K. (2018). Was ist interkulturelles Coaching? 20 Jahre und (k)ein bisschen Klarheit. Organisationsberatung, Supervision, Coaching, 25(1), 21-39. https://doi.org/10.1007/s11613-018-0532$\mathrm{x}$. 
Nazarkiewicz, K., \& Krämer, G. (2012). Handbuch Interkulturelles Coaching. Konzepte, Methoden, Kompetenzen kulturreflexiver Begleitung. Göttingen: Vandenhoeck \& Ruprecht.

Ong, Y.L., Bannon, M., \& Paice, E. (2004). Getting refugee doctors back to work : challenges, obstacles and solutions. In N. Jackson \& Y. Carter (Hrsg.), Refugee doctors: support, development and integration in the NHS (S. 31-37). Abindon: Radcliffe.

Pütz, R., Kontos, M., Larsen, C., Rand, S., \& Ruokonen-Engler, M. K. (2019). Betriebliche Integration von Pflegefachkräften aus dem Ausland. Innenansichten zu Herausforderungen globalisierter Arbeitsmärkte. https://www.boeckler.de/pdf/p_study_hbs_416.pdf. Zugegriffen: 7. Apr. 2020.

Sakamoto, I., Chin, M., \& Young, M. (2010). "Canadian experience," employment challenges and skilled immigrants. A close look through "tacit knowledge". Canadian Social Work, 12(1), 145-151.

Schroll-Machl, S., Schmid, S., \& Slate, E. (2018). Interkulturelles Coaching - eine komplexe, systemische Herausforderung. Organisationsberatung, Supervision, Coaching, 25(1), 41-57. https://doi.org/10. 1007/s11613-018-0535-7.

Stewart, E. (2003). A bitter pill to swallow: obstacles facing refugee and overseas doctors in the UK'. New issues in refugee research. Working Paper No. 96. United Nations High Commissioner for Refugees: Switzerland. https://www.unhcr.org/research/working/3fbb94a32/bitter-pill-swallowobstacles-facing-refugee-overseas-doctors-uk-emma-stewart.html. Zugegriffen: 7. Apr. 2020.

Willott, J., \& Stevenson, J. (2013). Attitudes to employment of professionally qualified refugees in the United Kingdom. International Migration, 51(5), 120-132. https://doi.org/10.1111/imig.12038.

Yin, R. K. (2014). Case study research: design and methods (5. Aufl.). Los Angeles: SAGE.

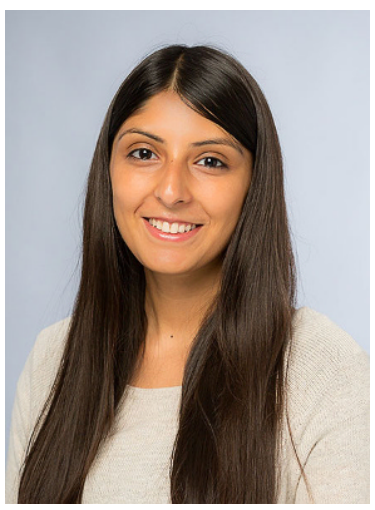

Sidra Khan-Gökkaya hat Kultur- und Religionswissenschaft, Internationale Migrationsforschung und Interkulturelle Beziehungen studiert; Integrationsbeauftragte am Universitätsklinikum Hamburg-Eppendorf, sie promoviert zum Thema „Berufliche Integration von geflüchteten Personen in Gesundheitsberufe“. Systemische Beraterin, Tätigkeit im Kontext von Diversität, Antidiskriminierung und Empowerment.

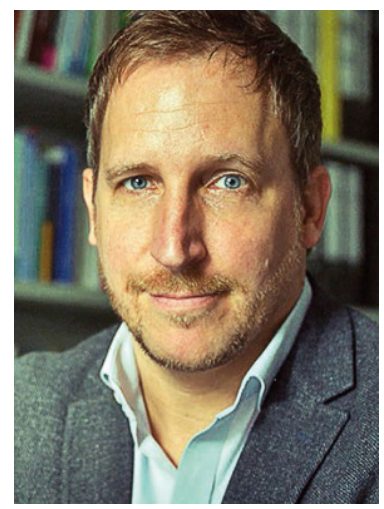

Prof. Dr. Mike Mösko Psychologischer Psychotherapeut, Leiter der Arbeitsgruppe Psychosoziale Migrationsforschung (AGPM) am Universitätsklinikum Hamburg Eppendorf. Forschungsschwerpunkte: Migration und Gesundheit, Mehrsprachigkeit und Interkulturelle Öffnung. Professor für Klinische Psychologie an der Hochschule MagdeburgStendal, Vorsitzender des gemeinnützigen Vereins SEGEMI (Seelische Gesundheit, Migration und Flucht e. V.), Hamburger Landessprecher der Deutschen Gesellschaft für Verhaltenstherapie. 